\title{
PD21 - Diagnosis and treatment of eosinophilic esophagitis guided by micro-arrays technology
}

\author{
Alicia Armentia1*, Jesus Barrio², Blanca Martin', Sara Martin , Alejandro Sanchez ${ }^{1}$, Alejandro Alvarez-Hodel', \\ Maria Jose Perez-Velesar ${ }^{1}$ \\ From 3rd Pediatric Allergy and Asthma Meeting (PAAM) \\ Athens, Greece. 17-19 October 2013
}

\section{Background}

Eosinophilic esophagitis (EoE) is an immune-mediated disease of the esophagus characterized by symptoms related to esophageal disfunction and histollogically by an eosinophil-predominat inflammation. Multiple therapies have been suggested to be helpful in EoE including endoscopic dilation, medical therapy and withdrawal dietary but, the election of what food should be excluded is very difficult.

Component-resolved diagnosis and microarray technology have been recently introduced into clinical allergy practice, and may be particularly useful in food-sensitized allergic patients.

\section{Methods}

We studied 67 patients suffering from EoE diagnosed by clinical symptoms and endoscopic biopsy esophageal with $>15$ eosinophils/high-powered field. Microarray technique (Thermofisher scientific), including detection of 112 allergens were performed in these 67 patients, in 50 allergic controls with pollen sensitization but without digestive symptoms, and 50 healthy people.

\section{Results}

Only 7 of the 67 patients that suffered from EoE did not present any allergen sensitization. All control patients presented sensitization to different pollen allergens without any predominant allergen. Nevertheless, among the patients with EoE and with response to any allergen, the predominant were $\mathrm{nCyn} \mathrm{d} 1$ (Cynodon dactylon or Bermuda grass pollen) $59.5 \%$, and the following allergens: Lipid transfer proteins (LTPs) from peach (19.40\%), hazelnut $17.91 \%$ and mugwort $19.40 \%$. Profilins were positive in $9.5 \%$ of the patients. Among nuts, allergens from hazelnut and walnut (21.4\%) were the most important. Other food allergens as Anisakis, egg or milk, only were positive in $9.5 \%, 2.3$ and $4.7 \%$ respectively.

\section{Conclusions}

High sensitization to vegetable allergens is relevant among patients with EoE. The most important implicated allergens are LTPs (usually associated to severe allergic response) from nuts and fruits and antigen 1 from Cynodon dactylon. Our patients are being treated with exclusion of the implicated food and pollen specific immunotherapy with preliminary favourable results.

\section{Authors' details}

${ }^{1}$ Allergy Unit, Rio Hortega University Hospital, Valladolid, Spain.

${ }^{2}$ Gastroenterology Service, Rio Hortega University Hospital, Valladolid, Spain.

${ }^{3}$ Pediatrics Service, Rio Hortega University Hospital, Valladolid, Spain.

Published: 28 February 2014

doi:10.1186/2045-7022-4-S1-P21

Cite this article as: Armentia et al.: PD21 - Diagnosis and treatment of eosinophilic esophagitis guided by micro-arrays technology. Clinical and Translational Allergy 2014 4(Suppl 1):P21.

${ }^{1}$ Allergy Unit, Rio Hortega University Hospital, Valladolid, Spain

Full list of author information is available at the end of the article 